\title{
Retos y oportunidades para el estudio, manejo y gestión del patrimonio industrial en Colombia
}

\author{
Challenges and opportunities for the study and \\ management of industrial heritage in Colombia
}

\section{Resumen}

Autores:

Natalie Rodríguez-Echeverry* rodriguezn@javeriana.edu.co

Carlos Del Cairo-Hurtado** carlos.delcairo@uexternado.edu.co

Laura Victoria Báez-Santos** victoriabaezsantos@gmail.com

Jesús Alberto Aldana-Mendoza** jesus.aldana@est.uexternado.edu.co

* Pontificia Universidad Javeriana ** Universidad Externado de Colombia

Colombia

Recibido: 16/Mar/2021 Aceptado: 26/Jun/2021
E I impacto producido por los grandes avances industriales en diferentes partes del mundo se manifestó en distintos contextos de Colombia que adaptaron y/o transformaron el espacio para la producción en masa de los diversos bienes utilizados por la sociedad. En consecuencia, el Patrimonio Industrial surgió de la necesidad de valorizar dichas manifestaciones materiales e inmateriales, como memorias de la industrialización. El objetivo del presente artículo de revisión crítica radica en discutir alrededor de los retos y las oportunidades que ofrece el Patrimonio Industrial en Colombia para su estudio, manejo y gestión. Esto, por un lado, a partir de un análisis del estudio de este patrimonio en Colombia, abordando las aproximaciones a la materia, así como las perspectivas y oportunidades recopiladas desde las áreas del conocimiento. Por otro lado, reflexionando sobre el devenir de este patrimonio y discutiendo algunas propuestas locales para su manejo y gestión. No obstante, la conservación de este patrimonio se encuentra sometida a complejos desafíos debido a las presiones sociales de diversas índoles que lo rodean, así como a la ausencia de políticas culturales sólidas de protección.

Palabras clave: patrimonio industrial; retos y oportunidades; manejo y gestión cultural; inventario co-participativo; Colombia.

\section{Abstract:}

The impact produced by the great industrial advances in different parts of the world was manifested in different contexts of Colombia that adapted and / or transformed the space for the mass production of the various goods used by society. Consequently, Industrial Heritage arose from the need to value these material and intangible manifestations, as memories of industrialization. The objective of this critical review article is to discuss the challenges and opportunities that Industrial Heritage offers in Colombia for its study and management. This, on the one hand, from an analysis of the study of this heritage in Colombia, addressing the approaches to the matter, as well as the perspectives and opportunities collected from the areas of knowledge. On the other hand, reflecting on the future of this heritage and discussing some local proposals for its management. However, the conservation of this heritage is subject to complex challenges due to the social pressures of various kinds that surround it, as well as the absence of solid cultural protection policies.

Keywords: industrial heritage; challenges and opportunities; cultural management; co-participative inventory; Colombia. 


\section{Introducción}

El surgimiento de la Revolución Industrial produjo grandes cambios a escalas nunca antes imaginadas en la historia de la humanidad. Estas transformaciones, que se expanden a las distintas esferas culturales, políticas, económicas y sobre todo tecnológicas de las sociedades, tuvieron un gran impacto en las fases productivas para la elaboración de elementos de uso cotidiano. La adquisición de la materia prima, su transporte, transformación, ensamblaje y conversión en un artefacto aumentó drásticamente gracias a los avances tecnológicos registrados en diferentes partes del mundo (Cannadine, 1985; Berg, 1995).

De forma paralela, al territorio colombiano también llegaron aquellos efectos consecuencia de la producción a gran escala de bienes de distintas índoles. Por ejemplo, en Bogotá las fábricas de principios del XIX no eran más que telares, molinos y talleres artesanales ubicados en las casas, o en lugares lejanos a la ciudad, como era el caso de los chircales y tejares existentes desde el siglo XVI (Escovar, 2004). Esta tendencia se mantuvo hasta la década de 1840 , cuando algunos extranjeros decidieron montar algunas fábricas de vidrio, papel o cristalería. Estas, pese a todos los esfuerzos, fracasaron en medio de una sociedad cuyas necesidades no eran las mismas de Europa en la industrialización (Escovar, 2004).

A pesar de este tímido proceso de industrialización, el espacio se transformó; se edificaron nuevas estructuras y se produjeron objetos que manifestaron un evidente impacto para el mundo, en sus planos naturales $y$ sociales. El inicio de nuevas prácticas y la presencia de nuevos escenarios conllevó, con el pasar de los años, a la configuración de un Patrimonio Industrial. Respecto a los elementos tangibles, las distintas actividades inmiscuidas en la producción industrial dieron como resultado contextos materiales e inmateriales que, en muchas ocasiones, se lograron preservar hasta la actualidad. Numerosos elementos se perdieron, algunos se transformaron y otros perduraron con el pasar del tiempo para que puedan ser estudiados desde las diferentes perspectivas que ofrecen las disciplinas interesadas por el patrimonio y su trascendencia para el presente (Therrien, 2008; Beltrán-Beltrán, 2008).

El Patrimonio Industrial resulta siendo un legado arqueológico, arquitectónico e histórico que puede aportar una gran cantidad y diversidad de información sobre aquellas dinámicas acontecidas en el territorio, sujetas a los cambios producidos por la industrialización (Benito del Pozo, 2002; Beltrán-Beltrán, 2008; Santa, 2018a). Así, es posible plantear y responder interrogantes vinculados no solo a la producción de los elementos, sino también a su uso e influencia en las poblaciones colombianas. Por lo tanto, el análisis del Patrimonio Industrial no se debe limitar al estudio de los espacios en donde se elaboraban los elementos. Por el contrario, es necesario que se aborden aspectos vinculados a las prácticas sociales de producción y consumo, así como el resultado de los bienes que terminaron depositados en variados espacios en el territorio. De igual manera, es necesario crear investigaciones y políticas co-participativas que logren establecer vínculos entre la academia, las instituciones y las comunidades.

Así como cualquier patrimonio cultural, el Patrimonio Industrial no está exento de factores que, de una u otra manera, han afectado considerablemente su preservación con el pasar de los años (Santa, 2018a). De hecho, a pesar de su histórica presencia extendida a nivel regional, muchos de sus elementos se han perdido de manera irremediable (Therrien, 2008). En definitiva, este patrimonio resulta siendo considerablemente particular (dada sus escalas espaciotemporales y las dinámicas que produjo en las poblaciones locales), en relación con los demás contextos culturales (prehispánicos, históricos, republicanos o contemporáneos) del territorio colombiano.

De ahí la necesidad de establecer nuevas perspectivas de análisis y protección, para garantizar que los contextos industriales que aún se preservan puedan salvaguardarse para las generaciones venideras. Partiendo de lo anterior, el principal propósito de este artículo consiste en plantear una discusión alrededor de aquellos retos y oportunidades que brinda el Patrimonio Industrial para garantizar su óptimo estudio, manejo y gestión $n^{1}$; en primer lugar abordando el estudio que se ha planteado alrededor de este patrimonio en Colombia desde las diferentes áreas del conocimiento, y en segundo lugar discutiendo el devenir de lo industrial a partir de algunas propuestas que pueden contribuir a su manejo y gestión.

Desde una perspectiva conceptual y teórica, una de las maneras de entender este patrimonio es desde una arqueología de la industria, la cual consiste en el instrumento de conocimiento científico del conjunto de la cultura material, por lo cual contribuye a la construcción del patrimonio (Damien, 2006). Sin embargo, la arqueología industrial ha trascendido el campo de la cultura material y ha abordado enfoques antropológicos e históricos, en los que ya se tienen en cuenta la trayectoria de actitudes del trabajo, lenguajes propios de las labores, saber hacer y oralidad. En suma, es necesario comprender la historia de las técnicas que son movilizadas para explicar e interpretar algunas trazas del equipamiento, la organización del trabajo y la disposición de los espacios (Damien, 2006)

A través de la cultura material, la arqueología industrial se ha enfocado en el estudio de inmuebles, máquinas, fuentes de energía, materias primas, medios de

Esta reflexión surge en las discusiones generadas en los grupos de estudio Transiciones Territoriales de la línea de investigación Patrimonio, Territorio, Espacio y Poder, de la Universidad Javeriana, y Arqueología Industrial, de la Universidad Externado, en el marco de los resultados de la investigación "Patrimonio Industrial en Colombia: Inventario Cartográfico de la Cultura Material - Fase exploratoria". 
transporte, barrios obreros, así como la organización económica y social. Dicha materialidad se configura en un territorio, donde ciertos aspectos medioambientales son determinantes para la transformación y la configuración de un paisaje industrial (Symonds y Casella, 2006) Este último, entendido como la expresión de múltiples relaciones que se configuran entre los individuos, la sociedad y un territorio definido (Therrien, 2007b). En este sentido, se parte de la agrupación de significaciones socioculturales que existen gracias a la forma en la que un entorno físico es percibido por una o varias personas, por el testimonio de las dinámicas históricas entre e humano y su medio ambiente, y/o por la especificidad de las culturas, sus tradiciones, prácticas y creencias (Therrien, 2007b).

Por lo tanto, los paisajes industriales pueden estar comprendidos por las rutas, medios de transporte, canales de riego, inmuebles de empaque, campamentos, sistemas de acueducto, zonas de cultivo, fuentes naturales y de energía, entre otros (Cerdá, 2009). Así, todo esto se comprende desde el emplazamiento y la distribución de estos componentes en un territorio, logrando entender los límites de dicho paisaje industrial y de la producción (Symonds y Casella, 2006).

En este sentido, se reclama especial atención a los siglos recientes, que marcaron cambios radicales en las estructuras sociales políticas y económicas. Transformaciones tales como la tecnificación en el siglo XIX, la nueva concepción del trabajo y las tensiones en el seno de un nuevo orden social independista; aspectos que contribuyeron a importantes transformaciones que pueden evidenciarse en la cultura material, su distribución espacial y una manera distinta de apropiación del territorio. Con el fin de estudiar dichos procesos, las aproximaciones sociales a lo industrial han considerado aspectos relacionados con poder, inequidad, relaciones laborales, formación de clase y aspectos sociales de intercambio de recursos, además de perspectivas para entender estrategias y mecanismos de producción, mercado y consumo en el mundo moderno (Damien, 2006; Symonds y Casella, 2006).

\section{El estudio del Patrimonio} Industrial en Colombia: Aproximaciones a la materia, perspectivas y oportunidades desde las áreas del conocimiento

El Patrimonio Industrial en Colombia se vislumbra como campo de estudio vinculado a la indagación, protección, conservación, gestión y manejo del patrimonio cultural. Si bien la legislación colombiana no refiere ni trata lo industrial como nivel o tipo de patrimonio, los bienes inmuebles y muebles asociados, los grupos humanos vinculados, los paisajes resultantes, entre otros, están siendo analizados desde ámbitos investigativos, e intervenidos desde la práctica profesional. En este sentido, la exploración realizada evidencia olvidos y vacíos, pero también la relevancia y el interés por su intervención y conservación que aluden a la pertinencia de su abordaje como campo de indagación y acción; retos y oportunidades para la inclusión, manejo y gestión de lo industrial como parte del Patrimonio Cultural de la Nación.

Pese a no identificarse el Patrimonio Industrial como campo específico de abordaje desde lo estatal, algunas de sus representaciones se incluyen en la clasificación del patrimonio arquitectónico. Así, para el máximo ente de manejo y control del patrimonio colombiano, los bienes industriales se asocian al Grupo Arquitectónico aludiendo a la Arquitectura para la industria, asociándose a la producción de bienes y servicios, y distinguiéndose elementos como hornos, ferrerías, fábricas, etc. (Ministerio de Cultura, 2005). Igualmente, se referencian a la Arquitectura para el Transporte, definidas como "construcciones que son parte del equipamiento, destinado a prestar servicios de movilidad de carga y pasajeros", como las estaciones del ferrocarril, aeropuertos, terminales, etc. (Ministerio de Cultura, 2005, p. 58). Asimismo, se vinculan las Obras de Ingeniería como "hechos construidos que se han realizado para beneficio de la actividad humana del territorio, haciendo uso de la tecnología y, con ello, generando una transformación en el paisaje natural", destacándose puentes, túneles, muelles, acueductos, etc. (Ministerio de Cultura, 2005, p. 58).

Estas catalogaciones y aproximaciones desde lo arquitectónico restringen las calificaciones y significaciones patrimoniales, ya que privilegian lo físico, centrándose en la detección de atributos constructivos, estéticos y espaciales, lo que conlleva a desconocer relaciones vinculadas a bienes y conjuntos, a dinámicas sociales y de producción, así como interrelaciones espaciales con los contextos y territorios. Ahora bien, avances en el manejo y protección del patrimonio colombiano dan cuenta de declaratorias de inmuebles que reconocen valores $y$ atributos vinculados con lo industrial (Figura 1). Todos estos son elementos arquitectónicos que, aunque reconocen muestras industriales, no evidencian el amplio espectro a nivel del territorio colombiano, privilegiando las estructuras férreas sobre otras formas y ejemplos industriales también relevantes.

Para ejemplificar, el Decreto 746 de 1996 declara el conjunto de estaciones del ferrocarril como Bienes de Interés Cultural. Entonces, se les reconocen valores estéticos asociados con la unidad arquitectónica característica, valores históricos como medio de transporte de carga y pasajeros de mediados del siglo XX, y valores simbólicos como influenciador del desarrollo social, cultural y económico (Ministerio de Cultura, 2012). En suma, formas de valoración compartimentalizadas desconocen valores como el tecnológico, arqueológico y comunitario, entre otros, así como atributos materiales e inmateriales asociados a lo industrial. Sin embargo, estas declaratorias y valoraciones generalmente desconocen relaciones de conjunto, bienes asociados y procesos de estructuras anexas conformadoras de sistemas y complejos industriales. Así, destaca la implementación de formas de valoración y catalogación que perpetúan la invisibilización de procesos, significaciones, formas de producción, relaciones sociales, entre otros. De tal modo, 
se detectan tensiones entre las formas de aproximación al entendimiento del patrimonio cultural de la nación desde la visión estatal que pone como reto el entendimiento de lo industrial más allá de la arquitectura.

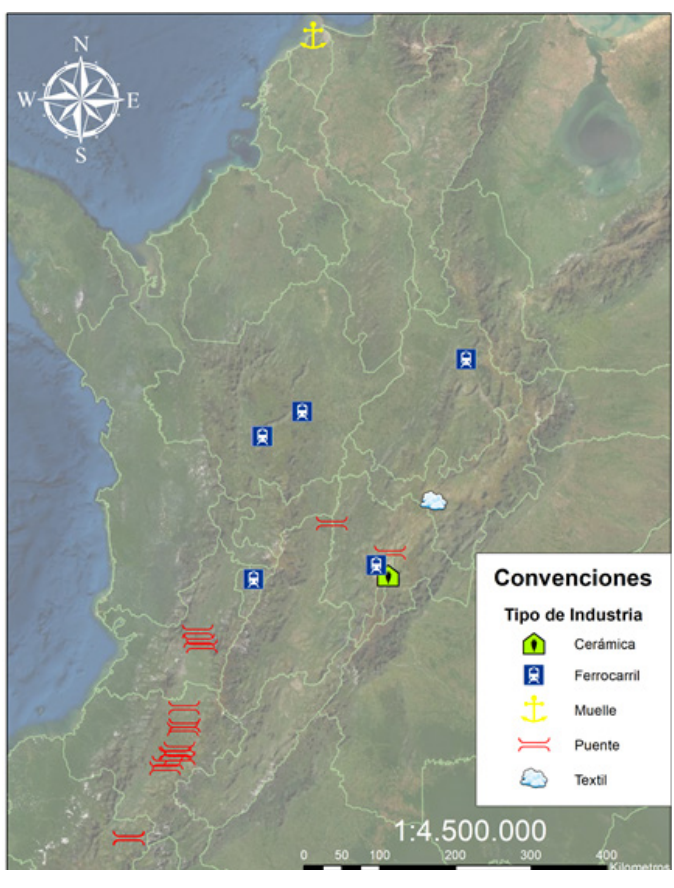

Figura 1: Bienes de Interés Cultural del Patrimonio Industrial en Colombia

Fuente: Elaboración propia (2021)
En lo relacionado con el manejo y la protección de los bienes patrimoniales de carácter industrial, se registran avances como la delimitación de Zonas de Influencia y la elaboración de Planes Especiales de Manejo y Protección (PEMP) de Bienes de Interés Cultural que buscan la protección y sostenibilidad en el tiempo. Así, se delimita la zona de influencia de 26 bienes y generan el Plan de Manejo y Protección de cuatro inmuebles industriales (Figura 2), lo que evidencia los adelantos obtenidos, pero a su vez el rezago respecto a su gestión. Ahora, si bien se delimitan áreas de afectación e influencia, estas no siempre se sustentan en valoraciones y entendimientos de los procesos asociados a los bienes, estructuras, interrelaciones funcionales, entre otros, que protejan los complejos industriales; convirtiéndose en límites y zonificaciones espaciales que desconocen diversos espacios, conexiones, construcciones y elementos asociados y constitutivos del Patrimonio Industrial.

En síntesis, se trata de formas de catalogación y valoración asociadas a las actividades industriales que privilegian unas representaciones formales y técnicas desconociendo otras. Asimismo, dispositivos de protección y manejo que relegan e invisibilizan bienes y prácticas asociadas a lo industrial; aproximaciones estatales que exponen a este patrimonio a un riesgo constante a desaparecer.

En contraposición, en los ámbitos académicos, se evidencia la promoción de lecturas que ponen al tema en discusión trazando perspectivas investigativas. La construcción de estudios de casos industriales ha brindado información de bienes, contextos, paisajes,

\begin{tabular}{|c|c|c|}
\hline BIEN DE INTERÉS CULTURAL & ZONA DE INFLUENCIA DEUMINATADA & $\begin{array}{l}\text { PLAN ESPECIAL DE MANEJO Y PROTECCIÓN } \\
\text { APROBADO }\end{array}$ \\
\hline Estación del Ferrocarril Cisneros & Resoludión 2236 del 4 de didembre de 2008 & \\
\hline Estación del Ferrocarril El Bosque & Resolución 2236 del 4 de díaembre de 2008 & \\
\hline Estadión del Ferrocarril Me dellín-Cisneros & Resolución 2236 del 4 de didembre de 2008 & \\
\hline Puente sobre el río Ovejas & Resolución 1941 del 3 de julio de 2015 & \\
\hline Puente sobre el río Pescador & Resolución 1941 del 3 de julio de 2015 & \\
\hline Puente sobre el río Piendamó & Resoludión 1941 del 3 de julio de 2015 & \\
\hline Puente del Humilladero & Resolución 1941 del 3 de julio de 2015 & \\
\hline Puente viejo sobre el río Cauca & Resolución 1941 del 3 de julio de 2015 & \\
\hline $\begin{array}{l}\text { Puente sobre el río Cauca en la vía entre } \\
\text { Popayán y Coconuco }\end{array}$ & Resolución 1941 del 3 de julio de 2015 & \\
\hline Puente real sobre el río Quilcacé & Resolución 1941 del 3 de julio de 2015 & \\
\hline Puente Los Libertadores & Resoludión 1941 del 3 de julio de 2015 & \\
\hline Puente sobre el río Timbío & Resolución 1941 del 3 de julio de 2015 & \\
\hline Puente sobre el río Cofre & Resolución 1941 del 3 de julio de 2015 & \\
\hline Puente sobre la que brada La Victoria & Resolución 1941 del 3 de julio de 2015 & \\
\hline Puente del Común & Resolución 019 de 1992 Zona influenda & \\
\hline Puente de los arcos sobre el río Juanambú & Resolución 1941 del 3 de julio de 2015 & \\
\hline Puente El Socorro sobre el río Juanambú & Resolución 1941 del 3 de julio de 2015 & \\
\hline $\begin{array}{l}\text { Conjunto Puente Natural Rumichacay } \\
\text { Antigua Casa de Aduana }\end{array}$ & $\begin{array}{l}\text { "El terreno comprendido entre la frontera con } \\
\text { Ecuador y un radio de } 100 \text { metros, partiendo del } \\
\text { centro de la Antigua Casa de Aduana") }\end{array}$ & \\
\hline Puente Navarro & Decreto 936 del 10 de mayo de 1994 (De dara) & \\
\hline Puente La Libertad sobre el río Guadalajara & Resolución 1941 del 3 de julio de 2015 (Dedara) & \\
\hline Puente sobre el río Sonso & Resolución 1941 del 3 de julio de 2015 & \\
\hline Puente sobre la que brada Popurrinas & Resolución 1941 del 3 de julio de 2015 (Dedara) & \\
\hline Estación del Ferrocarril Funza. (Demolida) & & Resolución 2452 del 18 de agosto de 2015 \\
\hline Estación del Ferrocarril Armenia & & $\begin{array}{l}\text { Resolución } 3277 \text { del } 17 \text { de octubre de 2014; } \\
\text { Resolución } 1230 \text { del } 19 \text { de mayo de } 2017 \\
\text { modifica y adiciona }\end{array}$ \\
\hline Estación del Ferrocarril Café Madrid & & Resolución 3594 del 14 de noviembre de 2014 \\
\hline Muelle de Puerto Colombia & Resolución 1223 del 26 de junio de 2009 & Resolución 1223 del 26 de junio de 2009 \\
\hline
\end{tabular}

Figura 2: Bienes de tipo industrial con mecanismos de manejo

Fuente: Elaboración propia a partir del Listado de Bienes declarados Bien de Interés Cultural, Ministerio de Cultura (2019) 
muestras representativas y ejemplos, avanzando en su documentación y valoración. Igualmente, se tiene la proyección de propuestas espaciales en bienes industriales que avanzan hacia la reflexión de conjuntos, paisajes y espacios, y en las formas de intervenirlos, manejarlos y gestionarlos. En definitiva, se observan esfuerzos investigativos que promueven la apertura hacia formas de reconocimiento y valoración del Patrimonio Industrial, desde disciplinas como la arquitectura, historia y arqueología, pero también apuestas interdisciplinares que propician abordajes integrales.

Esto ha permitido la generación de escenarios de sinergias entre áreas de conocimiento, instituciones y otros actores que simpaticen por la activación patrimonia de recursos culturales asociados a la industrialización. Desde la lectura patrimonial, la Facultad de Arquitectura y Diseño de la Pontificia Universidad Javeriana plantea formas de indagación asociadas con la cultura material inmueble, enfatizando en las relaciones asociadas con las estructuras industriales, la incidencia en la transformación de los paisajes urbanos y culturales, y la definición de criterios de valoración, a través de dos investigaciones: Hacia la Reflexión y Constitución de Patrimonio Industrial Colombiano y Patrimonio Industria Bogotano: Modelos y Paisajes Industriales.

Por su parte, el Programa de Arqueología de la Facultad de Estudios del Patrimonio Cultural de la Universidad Externado, viene realizando apuestas investigativas en torno al elemento industrial en relación con e componente de la arqueología pública y los complejos técnicos. Así se aúnan esfuerzos para desarrollar proyectos sobre paisajes industriales en Cartagena de Indias, tesis de grado en arqueominería de contextos industriales, así como el desarrollo de una asignatura de Arqueología Industrial que fomenta el interés por investigar desde la perspectiva arqueológica los contextos industriales.

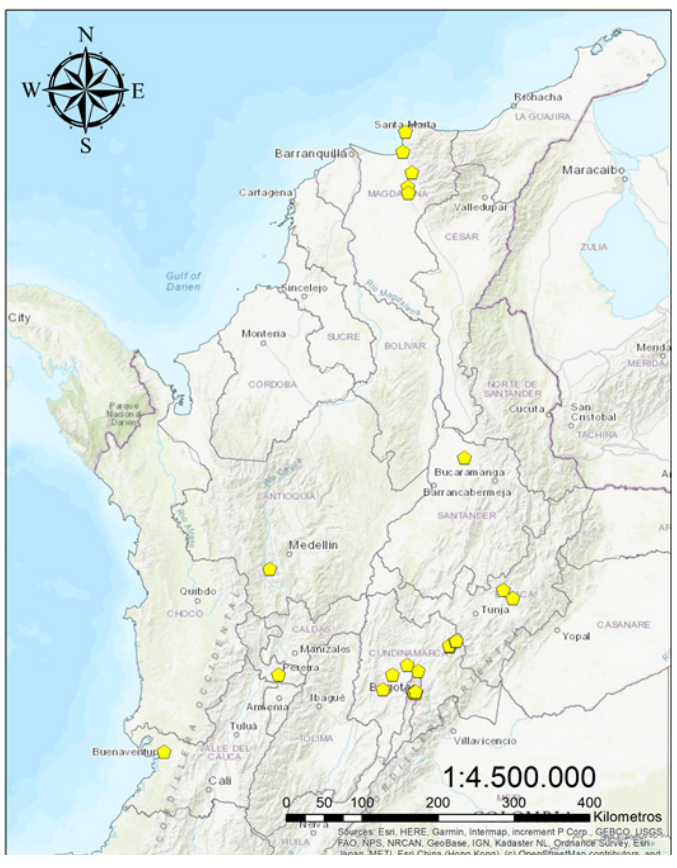

Figura 3: Investigaciones de la materialidad de Patrimonio Industrial en Colombia Fuente: Elaboración propia (2021)
Ahora, pese a los esfuerzos académicos que avanzan en la indagación industrial, en Colombia la aproximación a manejo, gestión e investigación del Patrimonio Industria ha sido relativamente incipiente. Sin embargo, se resaltan aproximaciones como los inventarios y propuestas de gestión de Patrimonio Industrial (Santa, 2018a; Álvarez 2011; Restrepo, 2013); las casas de las fincas cafeteras (Santa, 2018b); los molinos harineros del siglo XX (Pérez, 2018); los trabajos sobre los ferrocarriles (Arias de Greiff 2011), desde sus inicios (Poveda, 2002) hasta casos particulares en Pereira (Rivera, 2021a), y estaciones como la de Café Madrid en Bucaramanga (Rodríguez, 2020); el estudio de los complejos industriales en Barranquilla (Bell-Lemus, 2008), en Sabana de Torres (Rubiano, 2014), del Puerto Industrial Aguadulce S.A en Buenaventura (Gómez, 2014) y de la Fábrica de Loza de Bogotá (Therrien, 2007a). Recientemente se han planteado investigaciones académicas de diferentes universidades en contextos como Facatativá (Parada, 2020), Bucaramanga (Villamizar, 2019), Bogotá (Orbegozo, 2016; Malaver, 2020; Vargas, 1988), Samacá (Muñoz, 2014), Pereira (Rivera, 2021b), Cundinamarca y Boyacá (Pérez, 2018), Titiribí (Rodríguez, 2021) y La Mesa (Castillo, 2021) (Figura 3)

Respecto a los distintos aportes que se han formulado desde la arqueología para el contexto nacional, algunos estudios han logrado integrar los marcos teóricos y conceptuales esenciales de lo industrial. Así, en la Fábrica de Loza Bogotana, se abordaron aspectos relacionados a la producción, distribución y consumo de los objetos manufacturados en ella (Lamo y Therrien, 2001; Therrien, 2007a, 2008). Por su parte, Flórez (2009) también los discute en la identificación de los componentes que conformaban el sistema productivo de la fábrica de Tubos Moore en Bogotá. Por otro lado, Peña (2006) los aborda al realizar el rescate de los elementos producidos en la fábrica de lozas Faenza.

Posteriormente, Therrien y Del Cairo (2011) investigaron la Antigua Ferrería de Samacá y realizaron excavaciones del complejo industrial, analizando sus componentes muebles e inmuebles. En una escala más amplia e interdisciplinaria, Beltrán, Rodríguez y Therrien (2002; 2004) y Beltrán-Beltrán (2008) desarrollaron inventarios y estudios desde perspectivas analíticas sobre el Patrimonio Industrial en Bogotá y el Altiplano Cundiboyacense. Igualmente, Álvarez (2011) realizó una catalogación del Patrimonio Arqueológico Industrial de la zona central de Cundinamarca, con miras en alimentar el Atlas Arqueológico del Instituto Colombiano de Antropología e Historia.

Partiendo de todo esto, las investigaciones arqueológicas de lo industrial resultan ser apenas una tímida aproximación en el contexto nacional. A pesar de ser una subdisciplina naciente, surge la necesidad de comprender el universo de la cultura material, lo que permitirá a las nuevas generaciones identificar los tipos de sitios arqueológicos relacionados con la época de la industrialización. De esta manera, se busca construir estrategias que permitan clasificar y ordenar los objetos, estructuras y sitios del Patrimonio Industrial. El propósito, por lo tanto, es el de aproximarse a estos testimonios del proceso de industrialización bajo la idea de paisaje o de complejo, la cual, más que un inventario o suma 
de elementos, pasa a ser concebida como una unidad integrada para su posterior manejo y gestión.

\section{El devenir del patrimonio industrial colombiano: Pro- puestas para su manejo y gestión}

Como se ha observado, recientemente se puede advertir un creciente interés por el Patrimonio Industria en Colombia. A pesar de esto, se ha manifestado la necesidad de comenzar a desarrollar estrategias que busquen generar un fortalecimiento de este patrimonio en los diferentes niveles de su investigación, divulgación y gestión. Para este caso, se abordará el papel que pueden tener los inventarios de un Patrimonio Industrial. Así, se ha buscado generar una sistematización georreferenciada de los elementos vinculados al Patrimonio Industria mueble e inmueble, lo cual permite producir estrategias para su registro y estudio, y el establecimiento de redes de investigadores a nivel nacional e internacional. Para lograr llevar esto a cabo, es necesario obtener información que generalmente es recolectada en fichas de registro (o recursos similares) con las diferentes fuentes de información (Alfrey y Putnam, 2003).

Los inventarios sobre el Patrimonio industrial se convierten en herramientas que no solo son útiles como un recurso para el conocimiento, sino que además permiten tener una visión integradora de la cultura industrial. Entonces, sirven de base para determinar los niveles de conservación necesarios, aumentar e conocimiento e interés de las comunidades por su patrimonio, optimizar recursos técnicos, económicos, mano de obra, y generar alianzas interinstitucionales que atiendan a las necesidades del patrimonio (Alfrey y Putnam, 2003; Thornes, 1993). Por otro lado, permiten establecer diálogos entre la planeación urbana, turismo, empresas constructoras e instituciones que velan por la protección del Patrimonio Industrial (Parliamentary Assembly, 2013; Myers et al., 2013). De esta manera, son claves para establecer la viabilidad, planificación de los proyectos de gestión y seguimiento, comparación entre sitios, gestión ante riesgos y los proyectos de investigación alrededor de este tipo de sitios (Alfrey y Putnam, 2003; McCarthy, 2013; Stone, 2013). De igual forma, estas herramientas resultan siendo de suma importancia para las instituciones estatales en sus diversos procesos (por ejemplo, legislativos) necesarios para la protección, gestión y conservación de este patrimonio (Thornes, 1993).

En el ámbito internacional, para todo el patrimonio en general, mediante los Principios de Dublín se declara la necesidad de crear "inventarios integrados y listas de estructuras, sitios, áreas, paisajes, su entorno, objetos, documentos, dibujos y archivos asociados o patrimonio inmaterial" (ICOMOS y TICCIH, 2011), partiendo de la idea de que "toda persona y grupo social es creador de cultura y tiene derecho a formar parte y tomar parte de los procesos de reconocimiento, puesta en valor y transmisión del patrimonio" (Zabala et al., 2016).
Por consiguiente, se hace claro que es necesario establecer propuestas de registro que integren otras perspectivas y visiones para un entendimiento integral. Sin embargo, vale la pena aclarar, puede que en algunas ocasiones los inventarios se enfoquen en aspectos muy puntuales del Patrimonio Industrial. Por ejemplo, dejando de lado algunos de sus valores, concentrándose únicamente en los bienes inmuebles y sus características, o bien abordando los aspectos de conservación dados los propósitos con los que se realiza, convirtiéndose en un Directorio de Edificios (Alfrey y Putnam, 2003; Thornes, 1993). Esto sucede, generalmente, ya que se tiene una visión reducida desde el punto de vista de una disciplina en particular, desconociendo los conocimientos de las personas locales o los aportes de otras disciplinas, lo que a su vez genera la desvinculación entre la materialidad y su contexto arqueológico, arquitectónico, histórico, social, etc.

Por este motivo, se hace imprescindible la aplicación de planes orientados hacia la integración participativa de la totalidad de actores vinculados directa o indirectamente al patrimonio. Esto se realiza a partir de un proceso de coproducción del conocimiento, co-gestión y co-valoración patrimonial que permita establecer las relaciones culturales e identitarias entre los bienes culturales y los diversos actores que constituyen las poblaciones de territorio. De esta manera se parte de conceptos como ontologías múltiples, que tienen como propósito "integrar la emergencia de discursos y prácticas patrimoniales, observando una estrecha relación con la forma en que los sujetos imaginan dialécticamente su pasado y su futuro concibiendo el patrimonio según los ámbitos y ensamblajes con los que interactúe y se asocie" (Clavijo, 2021). Así, se parte de la idea de que el patrimonio surge a partir de las relaciones entre las diferentes experiencias de los autores y su instauración en la sociedad. Por ello, es posible concebir fundamentalmente dos modos de existencia: por un lado, el modo de subsistencia a través del cual "la forma patrimonial" logra mantener su forma material al mismo tiempo que es un remanente de pasado (tangible e intangible); y un modo de referencia dado que involucra diferentes formas de conocimiento y uso (González, 2015; Latour, 2007).

En este sentido, la propuesta de gestión y manejo de Patrimonio Industrial que se pretende desarrollar, surge de la necesidad de crear inventarios co-participativos $y$ dinámicos en los que se vinculen no solo profesionales de diferentes disciplinas e instituciones, sino también los conocimientos intrínsecos de las comunidades locales. Solamente de esta manera es posible horizontalizar los múltiples saberes que existen alrededor de este patrimonio, brindando nuevas estrategias para su protección en el corto y largo plazo. Por ende, se pretende fomentar un espacio en el que se produzca innovación, transmisión y coproducción de saberes con las comunidades. A través de este ejercicio, se busca destacar todos los valores que se le pueden llegar a otorgar al patrimonio, teniendo en cuenta que estos son aprehendidos y descubiertos por las personas, quienes han sido guiadas por sus contextos históricos, intelectuales, culturales y psicológicos (Lipe, 1990).

En definitiva, se busca una democratización de los saberes, donde las comunidades tengan el derecho 
a participar en la creación y transformación de conocimiento del patrimonio, adquiriendo un rol como constructores de memoria e identidades colectivas (Zabala et al., 2016). De esta forma, los inventarios coparticipativos contribuirían a crear un espectro más amplio de las investigaciones al permitir que discursos no-oficiales y saberes locales entren a dialogar con las diferentes disciplinas. Adicionalmente, se esperaría llegar a abarcar un mayor número de lugares o materialidad sobre el Patrimonio Industrial, al integrar aquellos que no estén reconocidos gubernamentalmente o en las fuentes históricas.

De igual manera, es una herramienta de apropiación social a partir de la cual pueden surgir más iniciativas en torno a la investigación, gestión o divulgación del patrimonio. Ejemplos de esto se pueden observar en los proyectos de ciudadanos científicos, en los cuales se busca que las comunidades participen en tres niveles: contribuidor, colaborador, co-creador (Rivera-Collazo et al. 2020). Para lograr este objetivo, el inventario debe integrar no solo los datos básicos de los complejos industriales, sino también un Sistema de Información Geográfica (SIG) colaborativo (Figura 4) en el que las personas tengan la posibilidad de brindar su propia información, a partir de sus diferentes vivencias o desde su memoria individual y colectiva.

Adicionalmente, esta propuesta pretende ser la base para la consolidación de nuevos valores, categorías y aspectos que determinen la gestión y protección a nive gubernamental, teniendo en cuenta que en Colombia el Patrimonio Industrial suele ser reconocido principalmente por sus características arquitectónicas. De esta forma, a partir de diálogos horizontales en los que se vincule a la academia, a las comunidades y a las instituciones se pueden crear nuevas categorías que resalten otro tipo de valores asociados a este patrimonio (más allá de lo tangible) (Figura 5). Esto, puede contribuir a la creación de políticas públicas o declaratorias de los contextos y paisajes industriales que incorporen valores nunca antes considerados, los cuales permiten una preservación integral de este patrimonio y de los elementos materiales (muebles e inmuebles) e inmateriales que lo componen.

Por este motivo, una de las primeras labores de esta propuesta consiste en la generación de un espacio propicio para la recopilación de la información arquitectónica, arqueológica, histórica y legal del Patrimonio Industrial. De este modo se resaltan no solo los elementos inmuebles que conformaron los diferentes sistemas productivos de extracción de materias primas, transporte, alimentación e infraestructuras, sino también aquellos elementos muebles que allí se encuentran; y al mismo tiempo abarcando también la relación de la cultura material con los paisajes interconectados, los conocimientos del saber-hacer, la organización del trabajo y el legado que moldea la vida de las comunidades que están vinculadas a estas infraestructuras, comprendiendo una amplia gama de aspectos que componen este patrimonio y sus manifestaciones culturales (ICOMOS y TICCIH, 2011).

Esta aproximación que integra las múltiples visiones, percepciones y representaciones en torno a la valoración patrimonial y la construcción colectiva del conocimiento industrial del territorio, se ha venido trabajando en proyectos como el de la aproximación patrimonial a las manifestaciones materiales e inmateriales del complejo ferroviario de Café Madrid (PEMP Café Madrid, 2012), el Estudio Arqueológico del Paisaje Industrial de la

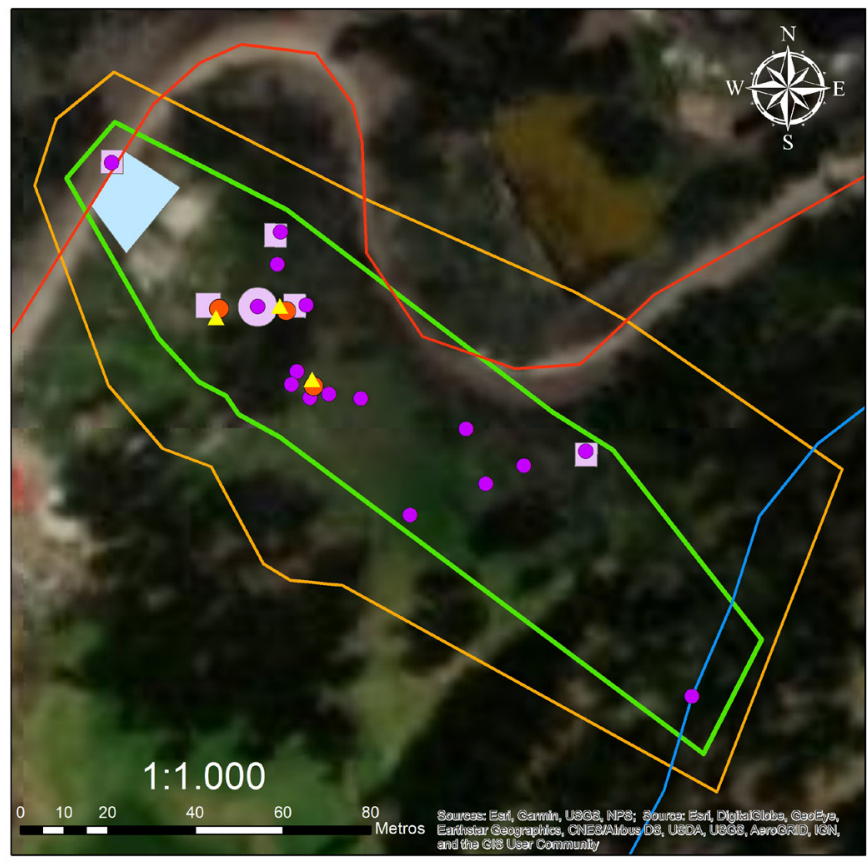

\section{Complejo de la Ferrería de Samacá} Modelo para la creación del Inventario de Patrimonio Industrial en Colombia

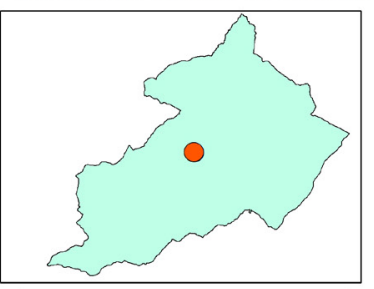

\begin{tabular}{|c|}
\hline Convenciones \\
$\square$ Fuentes de Materia Prima \\
$\square$ Planta de Procesamiento \\
$\square$ Transporte \\
\hline$\quad$ Fuentes de energia \\
Potencial Patrimonial \\
$\square$ Área Directa \\
$\square$ Area de Influencia \\
Bienes inmuebles \\
Bienes muebles \\
Intervenciones arqueológicas \\
Excavación \\
\hline
\end{tabular}

Figura 4: Propuesta de Sistema de Información Geográfica aplicada al contexto de la Ferrería de Samacá Fuente: Elaboración propia (2021) 


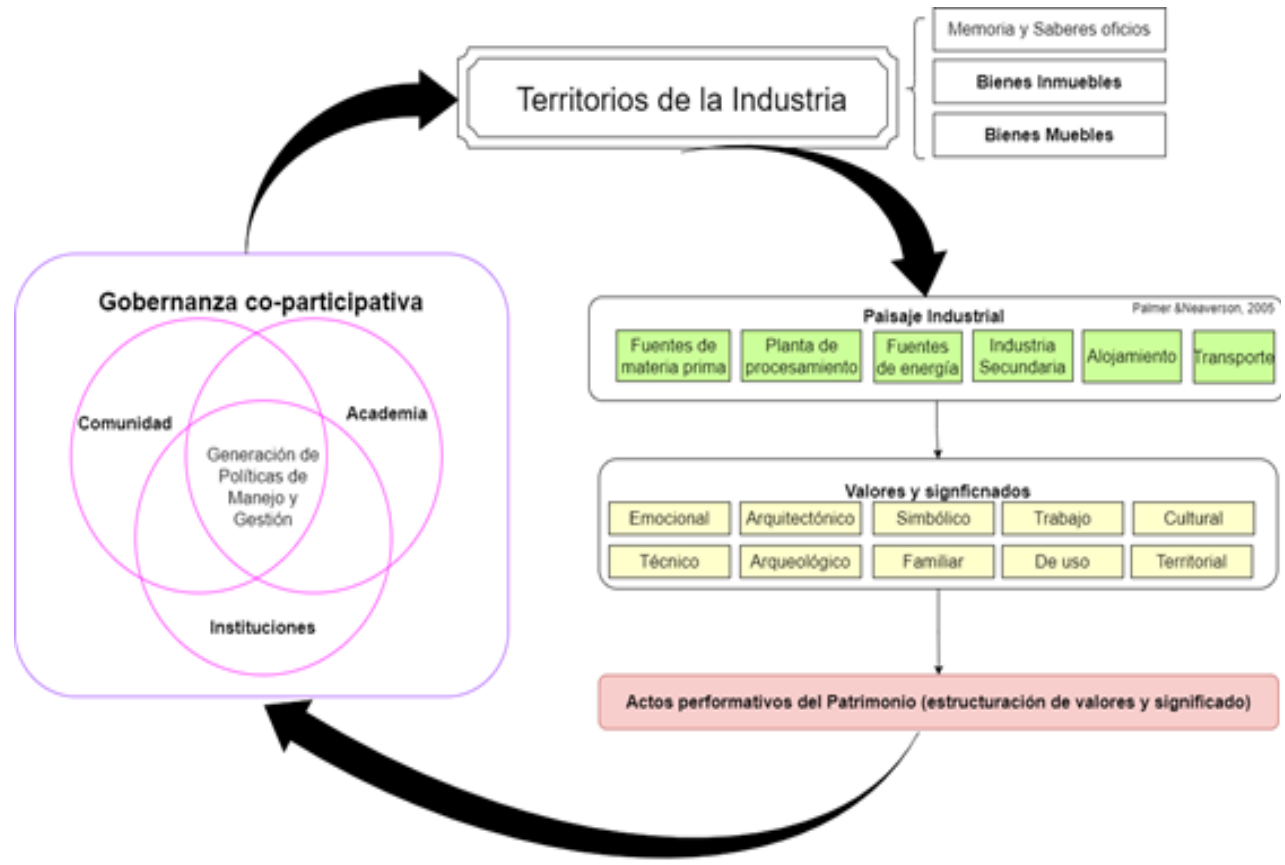

Figura 5: Diagrama propuesto para el estudio, manejo y gestión del Patrimonio Industrial Fuente: Elaboración propia (2021)

Antigua Ferrería de Samacá (Rodríguez et. al,. 2012), y la "Estrategia de Activación Patrimonial co-participativa en la Antigua Cervecería Andina del siglo XIX en Bogotá", que se ha venido desarrollando por el grupo Arqueología Industrial, de la Universidad Externado.

A partir de esto, otro énfasis que se debe realizar es fomentar la formación de personas interesadas por este patrimonio. En este caso, algunas iniciativas planteadas se han desarrollado en las Facultades de Estudios de Patrimonio Cultural (Universidad Externado), y de Arquitectura y Diseño (Pontificia Universidad Javeriana). Así se han generado escenarios de discusión sobre la importancia del estudio para el manejo y gestión de Patrimonio Industrial en el ámbito local, lo cual permitió proponer temáticas de reflexión en torno a las estrategias de valoración para garantizar la protección y conservación de los contextos industriales; y de igual manera reflexionar sobre las formas de inserción de los recursos patrimoniales de carácter industrial en el ordenamiento territorial a través de propuestas de refuncionalización, conjuntamente con la importancia de aproximarse a los bienes muebles como parte constitutiva de lo industrial.

Por otro lado, se ha buscado constituir una red de profesionales con capacidades para el estudio, manejo y gestión del Patrimonio Industrial, que puedan elaborar e implementar herramientas co-participativas y de estudios interdisciplinarios e interinstitucionales. Por consiguiente, se ha pretendido promover un escenario en torno a la gobernanza del Patrimonio Cultural Industria que busque construir y generar políticas públicas a partir de la concertación de miradas, perspectivas y proyecciones de los diversos actores involucrados en la gestión y manejo de los recursos culturales, suscitando una interacción horizontal de las diversas autoridades, locales y nacionales, con los actores locales de la sociedad civil y la empresa privada.

Finalmente, en el aspecto relacionado a la difusión del conocimiento, en 2020 se organizó un Seminario Internacional denominado Patrimonio Industrial y Perspectivas Interdisciplinares, que buscaba generar un espacio destinado a divulgar los estudios, exponer experiencias, intercambiar conocimientos y discusiones sobre esta temática, vinculando propuestas estudiantiles locales y aproximaciones investigativas de profesionales del ámbito nacional e internacional.

\section{Conclusiones}

El Patrimonio Industrial ha contribuido considerablemente a comprender los impactos sociales y naturales que produjo la llegada de la industrialización al continente. Ciertamente, este patrimonio se trata de un conjunto de bienes y prácticas que transformó las dinámicas de producción, distribución, consumo y uso de la cotidianidad colombiana. No obstante, como se pudo observar a lo largo del texto, este patrimonio aún es un campo de estudio reciente, el cual ofrece nuevas perspectivas de análisis, estudio y aproximación sobre los elementos que lo rodean. Únicamente a través de la integración de las diversas perspectivas, conocimientos, herramientas e instrumentos provenientes de las distintas disciplinas, instituciones y comunidades que rodean a este patrimonio, será posible construir $e$ implementar los procesos necesarios para garantizar un óptimo manejo y una apropiada gestión del Patrimonio Industrial en Colombia. 
De ahí la importancia de incorporar aproximaciones integrales al estudio de este patrimonio, incorporando los diversos valores y perspectivas arquitectónicas, arqueológicas y de visiones afines. Así, las descripciones, discusiones y reflexiones planteadas en el presente artículo permiten señalar los diferentes retos a los cuales se enfrenta el Patrimonio Industrial, ya que este no se encuentra exento de los múltiples desafíos a los que se enfrenta continuamente cualquier patrimonio. No obstante, al mismo tiempo, los estudios y perspectivas aquí reseñadas demuestran las oportunidades del Patrimonio Industrial y el rol que puede desempeñar en la comprensión de los espacios locales, comunitarios, regionales, nacionales, e incluso continentales.

En definitiva, el estudio, manejo y gestión de este patrimonio en Colombia ofrece diversos problemas $y$ al mismo tiempo, soluciones en el futuro próximo para los actores interesados en él. Uno de los principales problemas es la formulación de estrategias coparticipativas y dinámicas que integren las mencionadas nociones que rodean a lo industrial. El reto es lograr construir mecanismos que sean de las comunidades (con el apoyo de instituciones estatales y académicas) y para las comunidades; y que las nuevas generaciones sigan aportando las herramientas necesarias para que esto se lleve a cabo (lo cual parte de la formulación de espacios de formación y enseñanza).

Por ende, la propuesta del inventario co-participativo e integrador de perspectivas debe reflexionar más allá del registro, documentación, catalogación, georreferenciación y descripción técnica inmóvil y estática de los bienes. En realidad, se deben pensar nuevos procesos holísticos que se cuestionen y se preocupen por los bienes arquitectónicos, los objetos portables, los conocimientos que dieron lugar a la materialidad, las personas que actualmente interactúan constantemente con estos escenarios y los restos que aún existen, así como también los valores que configuran a este patrimonio. En suma, las aproximaciones nacionales al estudio del Patrimonio Industrial, las distintas perspectivas conceptuales y teóricas que existen de este, y las propuestas para su registro y protección, son estrategias que consideramos apropiadas para subsanar los retos y aprovechar al máximo las oportunidades para lograr un buen estudio, manejo y gestión de este patrimonio para Colombia.

En conclusión, la patrimonialización contribuye a generar efectos negativos para la dinámica social de ciertos grupos, ya que, en algunos casos, el patrimonio se convierte en una base de espectacularización, donde las manifestaciones culturales buscan satisfacer necesidades y expectativas a demandas particulares provocadas, sobre todo, por actores económicos. No obstante, a pesar de los aspectos negativos, es importante comprender la experiencia patrimonial como vehículo de memoria o de identidad, donde los objetos patrimoniales están dotados de valores considerados bajo criterios de historicidad, integridad y autenticidad, siendo el producto de un ejercicio de legitimación a través del estudio y diagnóstico de los bienes.

Por estos motivos, es de suponer la importancia de la integración de las formas alternativas de conocimiento sobre un contexto derivadas de la memoria, y el pasado de un contexto industrial por parte de los diversos grupos socioculturales. Ello permitirá encaminar los planes y estrategias patrimoniales donde prime la co-producción de conocimientos y la co-participación en el manejo y gestión de los recursos culturales. La apuesta, entonces, consiste en horizontalizar e integrar los discursos autorizados -institucionales y académicos- con los discursos disonantes -subalternos- (Smith, 2009). Es así como el patrimonio puede romper los límites impuestos por el orden institucional y académico, y puede entrar en un ámbito donde la sociedad civil atribuye otros valores tan legítimos como los valores políticos: el patrimonio es una experiencia física dotada de recuerdos a través del compromiso emocional. Por supuesto, esta es una idea que va en contra de la idea de pasividad mostrada en ciertos contextos, y resalta la importancia de la capacidad de agencia que permite una reacción y liberación de valores, relaciones y significados recíprocos.

Cómo citar este artículo/How to cite this article: Rodríguez-Echeverry, N., Del Cairo-Hurtado, C., Báez-Santos, L.V. y Aldana-Mendoza, J. (2021). Retos y oportunidades para el estudio, manejo y gestión del patrimonio industrial en Colombia. Estoa. Revista de la Facultad de Arquitectura y Urbanismo de la Universidad de Cuenca, 10(20), 117-127. doi: https://10.18537/est.v010.n020.a09 


\section{Referencias bibliográficas}

Alfrey, J. y Putnam, T. (2003). The Industrial Heritage: Managing Resources and Uses. Taylor y Francis.

Álvarez, A. (2011). Inventario y catalogación de Patrimonio Arqueológico Industrial de Colombia. Fase piloto-Zona Central de Cundinamarca: informe final. Instituto Colombiano de Antropología e Historia.

Arias de Greiff, J. (2011). Ferrocarriles en Colombia 1836 1930. Revista Credencial Historia, (257).

Bell-Lemus, C. (2008). Industria, puerto, ciudad (1870 1964). Configuración de Barranquilla. Apuntes. Revista de Estudios sobre Patrimonio Cultural, 21(1), 62-73.

Beltrán-Beltrán, L. (2008). Patrimonio industria colombiano: la definición de paisajes productivos en la Sabana de Bogotá. Apuntes. Revista de Estudios sobre Patrimonio Cultural, 21 (1), 26-43.

Beltrán, L., Rodríguez, N. y Therrien, M. (2002). Hacia la Reflexión y Constitución del Patrimonio Industrial Colombiano. Pontificia Universidad Javeriana.

Beltrán, L., Rodríguez, N. y Therrien, M. (2004). Patrimonio Industrial Bogotano: Modelos y Paisajes Industriales. Pontificia Universidad Javeriana.

Benito del Pozo, P. (2002). Patrimonio Industrial y Cultura del Territorio. Boletín de la Asociación de Geógrafos Españoles, (34), 213-227. https://bage.age-geografia.es/ ojs/index.php/bage/article/view/437

Berg, M. (1995). La era de las manufacturas, 1700-1820. Una nueva historia de la Revolución industrial británica. Mercados y manufacturas en Europa, Barcelona. Crítica.

Cannadine, D. (1985). El presente y el pasado en la Revolución industrial. Debats: Revista de cultura, poder i societat, (13) 74-94.

Castillo, N. (2021). Arqueología, Comunidad y Patrimonio Industrial: El Caso del Complejo Ferroviario de La Esperanza en La Mesa, Cundinamarca. Proyecto en curso. Universidad Externado de Colombia.

Cerda, M. (2008). Arqueología Industrial: teoría y práctica. Publicacions de la Universitat de València.

Clavijo, K. (2021). Propuesta de gestión co-participativa a través de la implementación de herramientas de arqueología pública para la puesta en valor y manejo del Área Arqueológica Protegida El Carmen (Usme). Universidad Externado de Colombia.

Damien, R. (2006). Archeologie industrielle, patrimoine industriel entre mots et notions. En Memoire de l'industrie de l'usine au patrimoine. Presse Universitaire De Franche-Comté.

Escovar, A. (2004). Atlas Histórico de Bogotá 1538- 1900 Editorial Planeta - Corporación la Candelaria.
Feliden, B. (2003). Conservation of historic buildings. Architectural press.

Flórez, D. (2009). Programa de monitoreo arqueológico para la adecuación de la carrera décima al sistema de Transmilenio, tramo no. 1. Informe final. Instituto Colombiano de Antropología e Historia.

Gómez, L. (2014). Programa de arqueología preventiva Puerto Industrial Aguadulce S.A - Buenaventura, departamento de Valle del Cauca: implementación plan de manejo arqueológico, fase de monitoreo vía de acceso al terminal portuario: informe final. Instituto Colombiano de Antropología e Historia.

González, P. (2015). Patrimonio y ontologías múltiples: Hacia la co-producción del patrimonio cultural. En C. Gianotti García, D. Barreiro Martínez, y B. Vienni Baptista (Eds.) Patrimonio y Multivocalidad. Teoría, práctica y experiencias en torno a la construcción del conocimiento en Patrimonio (pp. 179-198). Universidad de la República.

ICOMOS, y TICCIH. (2011). Dublin Principles -Joint ICOMOS- TICCIH Principles for the Conservation of Industrial Heritage Sites, Structures, Areas and Landscapes. ICOMOS.

Lamo, C. y Therrien, M. (2001). Loza fina para Bogotá: una fábrica de loza del siglo XIX. Revista de Antropología y Arqueología, 13, 199-228.

Latour, B. (2007). A Textbook Case Revisited. Knowledge as mode of existence. En J. E. Hackett, M. W. Lynch, J. Wajcman y O. Amsterdamska (Eds.). The Handbook of Science and Technology Studies (pp.83-112). MIT Press.

Lipe, W. (1990). Value and Meaning in Cultural Resources. En H. Cleer (Ed.) Approaches to the Archaeological Heritage. A Comparative Study of World Cultural Resource Management System, (pp. 1-11). Cambridge University Press.

Malaver, M. (2020). Patrimonio industrial: Una Forma de aproximarse al comienzo de la industria en Bogota a través del Estudio de la Antigua Cervecería Andina. Pontificia Universidad Javeriana.

McCarthy, D. (2013). Facing disaster: The importance of heritage inventories in preparation and response. Conservation perspectives. The GCl Newsletter, 28 (2), 16-17.

Ministerio de Cultura. (2005). Manual para Inventarios de Bienes Culturales Inmuebles. Ministerio de Cultura de Colombia.

Muñoz, L. (2014). Fábrica de hilados y tejidos de Samacá: patrimonio industrial colombiano. Proyecto de conservación. Centro de fomento y aprendizaje de prácticas tradicionales productivas sostenibles. Pontificia Universidad Javeriana.

Myers, D., Avramides, Y. y Dalgity, A. (2013). Changing the heritage inventory paradigm: The Arches open-source system. Conservation perspectives. The GCl Newsletter, 28 (2), 4-9. 
Orbegozo, J. (2016). Parque la Siberia: Hacia la reinterpretación y el reconocimiento del patrimonio industrial. Pontificia Universidad Javeriana.

Parada, T. (2020). Patrimonio Industrial de inicios del siglo XX en la sabana de Bogotá: Proyecto de restauración Molino San Carlos, Facatativá. Universidad Nacional de Colombia.

Parliamentary Assembly (2013). Industrial heritage in Europe. Pace - Resolution 1924. Parliamentary Assembly. Council of Europe.

PEMP Café Madrid. (2012). Plan Especial de Manejo y Protección (PEMP) de Bienes de Interés Cultural del Ámbito Nacional Estación Café Madrid ubicada en el Departamento de Santander. Consultoría n. 2436 de 2012. Ministerio de Cultura de Colombia.

Peña, G. (2006). Intervención arqueológica en el Teatro Faenza: Informe de actividades. Universidad Central, Instituto Colombiano de Antropología e Historia.

Pérez, M. (2018). Molinos harineros primera mitad del Siglo XX. Estrategias de gestión para la conservación del patrimonio industrial en Colombia [Tesis de Pregrado, Universidad de La Salle]. https://ciencia.lasalle.edu.co/ arquitectura/619/

Poveda, G. (2002). El primer ferrocarril en Colombia. Dyna, 69 (137), 61-73.

Restrepo, A. (2013). Patrimonio industrial y una propuesta para el Valle de Aburrá: Un gran patrimonio en el olvido. Apuntes. Revista de Estudios sobre Patrimonio Cultural, 26(2), 10-23

Rivera-Collazo, I. C., Rodríguez-Franco, C., Garay-Vázquez, J. J., Rivera-Claudio, H. M., y Estremera-Jiménez, R. (2020). Towards a definition and practice of communal archaeology: Ethics, informal learning, and citizen science in the practice of indigenous archaeology. Journal of Community Archaeology \& Heritage, 7(2), 120-134.

Rivera, T. (2021a). Redes férreas y patrimonio industria en la configuración urbano-territorial de Pereira: Ciudad intermedia del Paisaje Cultural Cafetero. Territorios, (44), 1-31. https://doi.org/10.12804/revistas.urosario.edu.co/ territorios/a.8319

Rivera, T. (2021b). De la fragmentación a la conectividad del paisaje cafetero: Una mirada sistémica al Área Metropolitana Centro Occidente y su patrimonio industrial y ferroviario. Proyecto en curso. Universidade Federal do Rio de Janeiro.

Rodríguez, N. (2020). Conformación de territorio y territorio de conformación: el caso del Conjunto Ferroviario de la Estación Café Madrid. Editorial Pontificia Universidad Javeriana.

Rodríguez, N., Cancino, J.C., Therrien, M., Del Cairo, C., Ballesteros, L. F., Ariza, W., Páez, V., Gutiérrez, G, López, M., Rico, E., Espinosa, G. (2012). Estudios Técnicos para la Restauración de los Buitrones de La Antigua Ferrería Municipio de Samacá - Boyacá. Alcaldía de SamacáGobernación de Boyacá.
Rodríguez, S. (2021). Arqueología industrial: un análisis de la minería de oro practicada en la Sociedad El Zancudo en el Siglo XIX, Titiribí, Antioquia. Proyecto en curso. Universidad Externado de Colombia.

Rubiano, J. (2014). Prospección arqueológica para la planta industrial Sogamoso, municipio de Sabana de Torres - Departamento de Santander: informe final. Instituto Colombiano de Antropología e Historia (ICANH).

Santa, A. (2018a). Inventario del patrimonio industrial bogotano. Arquitectura y Urbanismo, 39 (3), 64-76.

Santa, A. (2018b). La casa de las fincas cafeteras como patrimonio agroindustrial colombiano en riesgo. Revista Nodo, 12(24). 74-88. http://revistas.uan.edu.co/index. $\mathrm{php} /$ nodo/article/view/150

Smith, L. (2009). Intangible Heritage. Routledge.

Stone, P. (2013). War and heritage: using inventories to protect cultural property. Conservation perspectives. The GCl Newsletter, 28 (2), 13-15.

Symonds, J. y Casella, E. (2006). Industrial Archaeology Future Directions. Springer.

Therrien, M. (2007a). De fábrica a barrio: urbanización y urbanidad en la Fábrica de Loza Bogotana. Editorial Pontificia Universidad Javeriana.

Therrien, M. (2007b). Informe sobre el valor histórico o cultural de los objetos transportados en las embarcaciones hundidas en la época de la colonia. Instituto Colombiano de Antropología e Historia.

Therrien, M. (2008). Patrimonio y arqueología industrial: ¿lnvestigación vs. Protección? Políticas del patrimonio industrial en Colombia. Apuntes. Revista de Estudios sobre Patrimonio Cultural, 21 (1), 44-61.

Therrien, M. y Del Cairo, C. (2011). Estudio arqueológico antigua Ferrería de Samacá. Instituto Colombiano de Antropología e Historia.

Thornes, R. (1993). The changing shape of the inventory: New priorities and new approaches. En Architectura heritage: inventory and documentation methods in Europe (125-136). Council of Europe.

Vargas, C. (1988). Arquitectura ferroviaria en la Sabana de Bogotá [Tesis de Grado, Universidad de La Salle]. https://ciencia.lasalle.edu.co/arquitectura/2070

Villamizar, C. (2019). Complejo Patrimonial Café Madrid [Tesis de Grado, Pontificia Universidad Javeriana]. https:// repository.javeriana.edu.co/handle/10554/46212

Zabala, M., Fabra, M., Aichino, G. y De Carli, C. (2016). Reflexiones en torno a los aportes que realiza la Arqueología Pública en la construcción de memorias e identidades locales en el NE de la provincia de Córdoba (Argentina). E+E: Estudios de Extensión en Humanidades, 4 (4), 8-22. https://revistas.unc.edu.ar/index.php/EEH/ article/view/15086/15016 